\title{
Cloning of a DNA fragment encoding the 5 '-terminus of the botulinum type E toxin gene from Clostridium butyricum strain BL6340
}

\author{
Nobuhiro FujiI, ${ }^{1}$ Kouichi Kimura, ${ }^{1}$ Teruo Yashiki, ${ }^{2}$ TomokazU IndOH, ${ }^{1}$ \\ Tadayuki Murakami, ${ }^{1}$ Kayo TsuzUki, ${ }^{1}$ Noriko Yokosawa ${ }^{1}$ and KeIJI Oguma ${ }^{1 *}$ \\ ${ }^{1}$ Department of Microbiology, Sapporo Medical College, Sapporo 060, Japan \\ ${ }^{2}$ Laboratory of Technology, College of Medical Technology, Hokkaido University, Sapporo 060, Japan
}

(Received 9 July 1990; revised 1 October 1990; accepted 8 October 1990)

\begin{abstract}
Chromosomal DNA was extracted from toxigenic Clostridium butyricum strain BL6340 isolated from a case of infant botulism. After digestion by EcoRI, a DNA fragment of about $1 \mathrm{kbp}$ was cloned into Escherichia coli using $\lambda$ gt11, and was subcloned into pUC118. The $E$. coli cells transformed with this cloned fragment produced a $33 \mathrm{kDa}$ protein which reacted with monoclonal antibodies recognizing the light chain (Lc) component of botulinum type $E$ toxin. The nucleotide sequence of the cloned fragment was determined. The sequence was similar to that from botulinum type $\mathrm{E}$ toxin gene fragments previously determined by our laboratory (strains Mashike, Otaru and Iwanai). Several highly homologous sequences among the botulinum type $\mathrm{A}, \mathrm{C}, \mathrm{E}$, butyricum and tetanus toxin genes were found in both translated and untranslated regions. These results suggest that the toxin gene of $C$. butyricum may have evolved by transfer from $C$. botulinum.
\end{abstract}

\section{Introduction}

Clostridium botulinum is the causative agent of foodborne botulism or infant botulism. Neurotoxins produced by the bacteria are classified into seven groups (A to $G)$ based on their antigenicity. Recently, however, Clostridium species other than $C$. botulinum have been found to produce botulinum neurotoxin. $C$. butyricum and $C$. barati produce type $\mathrm{E}$ and type $\mathrm{F}$ neurotoxins, respectively, which are implicated in the onset of human infant botulism (Hall et al., 1985; McCroskey et al., 1986). The DNA extracted from a type E toxinproducing $C$. butyricum strain showed high homology to that obtained from a typical nontoxigenic $C$. butyricum strain (Suen et al., 1988). It is therefore important to establish whether toxin genes exist in toxigenic and nontoxigenic $C$. butyricum strains, and to clarify the mechanism of transfer of toxin genes from $C$. botulinum to other clostridia. The $\mathrm{N}$-terminal amino acid sequences of botulinum type A, B, C, D and E toxins, and of the toxin produced by toxigenic $C$. butyricum, have been determined (Gimenez et al., 1988; Moriishi et al., 1989;

Abbreviation: mAb, monoclonal antibody.

The sequence data in this paper have been submitted to EMBL and have been assigned the accession number X53180.
Sathyamoorthy et al., 1985; Tsuzuki et al., 1988). The 5'terminus and complete nucleotide sequence of type $A$ toxin gene has been reported (Betlay et al., 1989; Binz et al., 1990; Thompson et al., 1990). Recently, the complete nucleotide sequence of the structural gene of botulinum type $\mathrm{C}$ toxin and the 5 -terminal nucleotide sequence of the type E toxin gene (strains Mashike, Otaru, Iwanai) have been determined in our laboratory (Fujii et al., 1990; Kimura et al., 1990). The nucleotide sequences obtained from three type $\mathrm{E}$ toxin genes were identical. In this paper, we report the cloning, sequencing and expression of a toxin gene fragment from a toxigenic $C$. butyricum strain, and compare the $5^{\prime}$-terminal nucleotide sequence (and the deduced amino acid sequence) to those of botulinum toxins type $\mathrm{A}, \mathrm{C}$ and $\mathrm{E}$, and tetanus toxin.

\section{Methods}

Extraction of DNA from cells. One toxigenic strain of C. butyricum (BL6340, isolated from a case of infant botulism and kindly provided by Dr Hatheway, Centers for Disease Control, Atlanta, USA) and nine nontoxigenic strains (KZ145 and KZ589, provided by Dr Hatheway; IFO14810, IFO20064, Y-M-1a, Y-M-2a, Y-M-3a, Y-B-la and IAM19001, provided by Dr Arai, Biotherapy Research Institute, Tokyo, Japan) were used. These strains were cultured in $250 \mathrm{ml} \mathrm{LYG}$ medium $(1 \%(w / v)$ lactalbumin hydrolysate, $2 \%(w / v)$ yeast extract, 
$0.5 \%(\mathrm{w} / \mathrm{v})$ glucose, $0.15 \%(\mathrm{w} / \mathrm{v})$ cysteine hydrochloride, $\mathrm{pH} 7.2)$ at $30{ }^{\circ} \mathrm{C}$ for $12 \mathrm{~h}$. The cells were collected by centrifugation at 6000 r.p.m. $(11000 \mathrm{~g})$ for $10 \mathrm{~min}$ at room temperature, and resuspended in $20 \mathrm{ml}$ LYG medium containing $8 \%(w / v)$ polyethylene glycol $4000,10 \mathrm{U}$ penicillin $\mathrm{G} \mathrm{ml}^{-1}$ and $10 \mu \mathrm{g}$ lysozyme $\mathrm{ml}^{-1}$, and then incubated at $30^{\circ} \mathrm{C}$ for $60-90 \mathrm{~min}$. After centrifugation, the cells were lysed using 2-3\% $(w / v)$ of sodium dodecyl sulphate (SDS). Extraction and purification of chromosomal DNA were performed by the procedures described by Marmur (Marmur, 1961).

Monoclonal antibodies. Three monoclonal antibodies (mAbs) against light chain (Lc) component of type E toxin, EL161-38, EL211-3, EL219-15, were those prepared previously (Tsuzuki et al., 1988). EL211-3 and EL219-15 react only with type E toxin, but EL161-38 also reacts with Lc components of botulinum type B, C, D and tetanus toxins.

Cloning and sub-cloning of the toxin gene. Cellular DNA from toxigenic strain BL6340 was digested by EcoRI (Takara Shuzo Co., Kyoto, Japan), ligated to $\lambda$ gt11 EcoRI arms (Promega Corporation), and then packed in vitro by using Packagene (Promega Corporation). The gene library was plated on E. coli Y1090 (Promega Corporation), and positive clones were immunoscreened using mAb EL161-38 and the ProtoBlot Immunoscreening System (Promega Corporation). The isolated positive clones were purified, and the recombinant phage DNA was extracted. After digestion with EcoRI, the inserts separated from phage DNA were then sub-cloned into the EcoRI site on the polylinker of pUC118 (Takara Shuzo Co.).

DNA sequencing and Western blot analysis. DNA sequence analysis was performed by the dideoxy termination method using $\left[\alpha^{-35} \mathrm{~S}\right] \mathrm{dATP}$ (NEN Products) and a T7 DNA sequencing kit (Pharmacia).

Gene products from $E$. coli strain Y1090 infected with recombinant phage, and from E. coli strain MV1184 transformed by recombinant plasmids, were analysed by Western blots with mAbs and with alkaline-phosphatase-labelled anti-mouse immunoglobulin $\mathbf{G}$ conjugate (Promega Corporation), nitroblue tetrazolium and 5-bromo-4chloro-3-indolyl phosphate. The transformed cells were incubated in $10 \mathrm{ml} 2 \times \mathrm{TY}$ medium $[1.6 \%(\mathrm{w} / \mathrm{v})$ tryptone, $1 \%(\mathrm{w} / \mathrm{v})$ yeast extract, $0.5 \%(\mathrm{w} / \mathrm{v}) \mathrm{NaCl}, \mathrm{pH} 7.6]$ at $37^{\circ} \mathrm{C}$ for $12 \mathrm{~h}$. After centrifugation, the cells were suspended in $1 \mathrm{ml}$ TEP buffer $(100 \mathrm{~mm}-\mathrm{Tris} / \mathrm{HCl}, \mathrm{pH} 7 \cdot 4$, $10 \mathrm{mM}$-EDTA, $1 \mathrm{mM}$-PMSF), sonicated for about $2 \mathrm{~min}$ at $0^{\circ} \mathrm{C}$, and centrifuged at 12000 r.p.m. for $10 \mathrm{~min}$ at $4^{\circ} \mathrm{C}$. The resulting supernatants were electrophoresed on $12 \%(w / v)$ SDS-polyacrylamide gel, and Western blot analysis was carried out as described previously (Tsuzuki et al., 1988).

Dot blot hybridization. DNA samples $(2,20$ and $40 \mu \mathrm{g})$ from toxigenic and nontoxigenic strains of $C$. butyricum were spotted on a nylon membrane (Pall Ultrafine Filtration Corp., Glen Cove, NY, USA). After denaturation and neutralization, the membrane was prehybridized in $50 \%(\mathrm{v} / \mathrm{v})$ formamide hybridization buffer at $42^{\circ} \mathrm{C}$ for $12 \mathrm{~h}$, and then hybridized with ${ }^{35}$ S-labelled toxin probe using a DNA labelling kit (Nippon Gene, Toyama, Japan) at $42^{\circ} \mathrm{C}$ for $24 \mathrm{~h}$ according to the procedures described previously (Fujii et al., 1988). The membrane was washed at $68^{\circ} \mathrm{C}$ and autoradiographed.

\section{Results and Discussion}

\section{Cloning and gene products}

Chromosomal DNA isolated from the toxigenic $C$. butyricum strain BL 6340 was digested with EcoRI and ligated to $\lambda \mathrm{gt} 11$ EcoRI arms. After in vitro packaging,

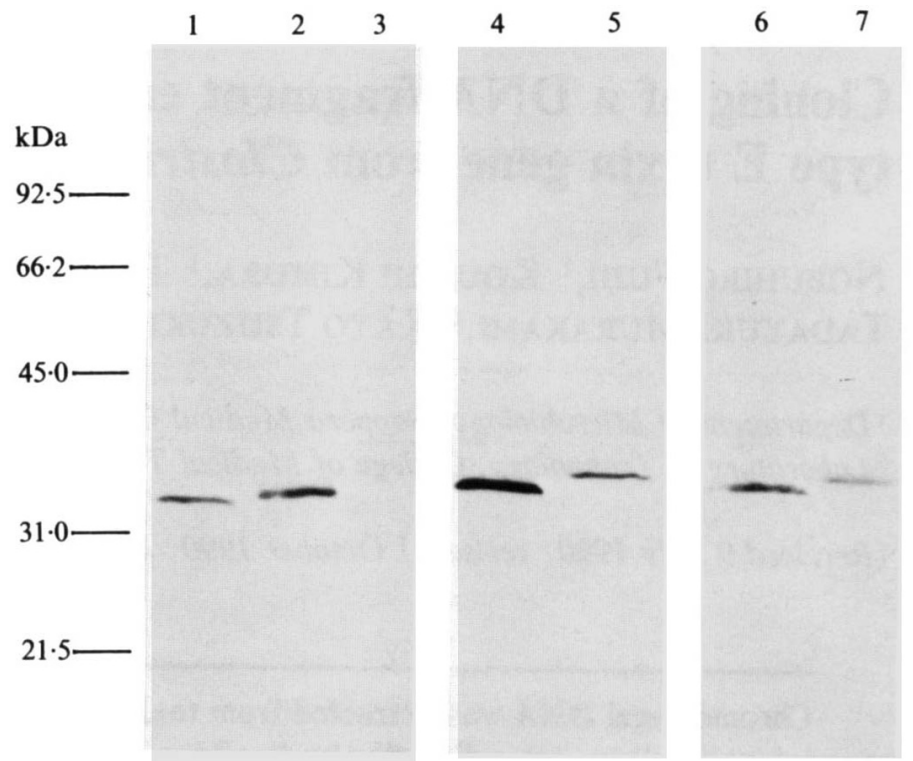

Fig. 1. Western blot analysis of gene products by recombinant phage and plasmid. Cellular extracts from $E$. coli Y1090 infected with (1) $\lambda$ gt 11 containing a $1 \mathrm{kbp} E c o R I$ fragment of the butyricum toxin gene ( $\lambda \mathrm{BU}-\mathrm{Ll})$; (2) $\lambda \mathrm{gt} 11$ containing a type $\mathrm{E}$ toxin gene EcoRI fragment (גEM-L1); (3) control $\lambda \mathrm{gt} 11$; and from $E$. coli MV1184 transformed by pUC118 containing butyricum toxin gene $E c o$ RI fragment (pBU-L1) (4 and 6) or botulinum type $\mathrm{E}$ toxin gene EcoRI fragment (pEM-L1) (5 and 7). The extracts were characterized by Western blots using mAb EL161-38 against botulinum type $E$ toxin light chain. The cells of lanes $1-5$, and lanes 6-7 were incubated in the presence and absence of IPTG, respectively.

and infection into $E$. coli, the recombinant plaques were screened for the production of toxin fragments using mAb EL161-38. Several positive clones were detected and one, $\lambda B U-L 1$, was chosen for further study. $\lambda B U-L 1$ DNA was prepared, and found to contain a single $1 \mathrm{kbp}$ $E c o$ RI insert. E. coli Y1090 cells infected with phage $\lambda B U-L 1$ produced a $33 \mathrm{kDa}$ protein which reacted with mAb EL161-38 both in the presence and in the absence of IPTG (Fig. 1). In addition, the infected cells reacted with mAb EL211-3, but not with mAb EL219-45 (data not shown). The $1.0 \mathrm{kbp}$ EcoRI fragment was isolated from $\lambda \mathrm{BU}-\mathrm{L} 1$, and then recloned into the EcoRI site of pUC118. E. coli strain MV1184, when transformed by the recombinant plasmid ( $\mathrm{pBU}-\mathrm{L} 1$ ) produced a protein of similar size $(34 \mathrm{kDa})$ as that obtained from the phage clone (Fig. 1). These gene products migrated slightly faster than those produced by $E$. coli cells that had been transformed with phage or plasmid expressing an analogous fragment (about $1 \mathrm{kbp}$ ) which encodes the botulinum type E toxin gene (Fujii et al., 1990).

\section{Susceptibility of insert to endonuclease}

The $1.0 \mathrm{kbp} E c o$ RI fragment from pBU-L1 was digested with several restriction enzymes, and the restriction map 
(a)

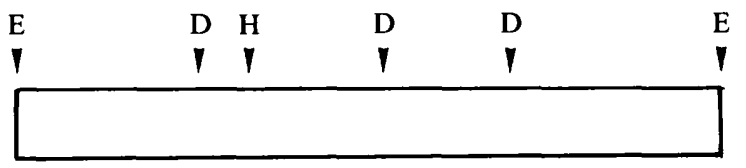

(b)

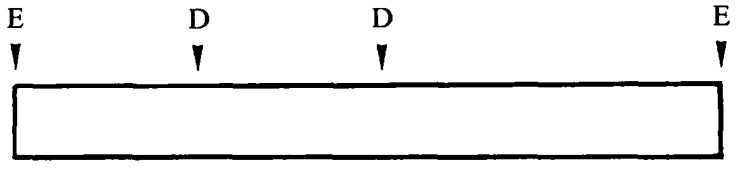

$\overline{100 b p}$

Fig. 2. Restriction endonuclease recognition sites in the fragments from $C$. butyricum $(a)$ and $C$. botulinum type $\mathrm{E}(b)$. The map was determined by $4 \%$ NuSieve agarose gel electrophoresis of the plasmid DNAs cleaved by EcoRI (E), DraI (D) and HincII (H)

was found to be similar, but not identical, to that of the analogous $1.0 \mathrm{kbp}$ fragment from the $C$. botulinum type $\mathrm{E}$ toxin gene (Fig. 2).

\section{Nucleotide and derived amino acid sequences of butyricum toxin gene EcoRI fragment}

The 5 -terminal nucleotide sequence $(756 \mathrm{bp})$ of the butyricum toxin gene and $227 \mathrm{bp}$ of the $5^{\prime}$-untranslated region was determined. The number of nucleotides of the coding and noncoding regions was the same as that of the cloned type E toxin gene fragment (Fujii et al., 1990). However, the nucleotide sequences of the butyricum and botulinum type $\mathrm{E}$ toxin genes were not identical; eight bases in the untranslated region, and 27 bases in the open reading frame were different (Fig. 3). A Shine-Dalgarno consensus sequence (AGGAGA) was found 14 nucleotides upstream from the translation initiation start codon (ATG). A base sequence corresponding to the -10 consensus region of $E$. coli (TATAAT) was identified at position 87 , but no sequence corresponding to the -35 consensus sequence of $E$. coli (TTGACA) was detected. Two stem-loop structures, at bases 17 to 48 and 121 to 155 , were also detected in the region upstream of the -10 region and the Shine-Dalgarno sequence, respectively. The deduced $\mathrm{N}$-terminal amino acid sequence of butyricum toxin was identical to that previously reported by protein analysis of the toxin (Gimenez et al., 1988). It was also similar to the protein sequence of the botulinum type E toxin (strains Mashike, Otaru and Iwanai) except for 17 amino acid residues, which were altered by the nucleotide changes described above (Fig. 4).
Comparison of nucleotide and amino acid sequences of the putative toxin gene with those of botulinum type $A, C$ and tetanus toxins

As described above, the nucleotide sequences of butyricum and type $\mathrm{E}$ toxin gene fragments were similar in both the noncoding and coding regions. Since the nucleotide sequences of types $\mathrm{A}, \mathrm{C}$ and $\mathrm{E}$, and tetanus toxin gene have been determined, the sequences of these five toxin genes were compared. There exist several regions of homologous sequence in both untranslated and translated regions (Fig. 3). In the untranslated region, it was very easy to find sequences corresponding to a Shine-Dalgarno sequence which is presumably involved in the initiation of translation of the toxin genes. In contrast, the sequence corresponding to the -10 and -35 regions was difficult to identify, due to the high A-T content of the DNA. However, one potential -10 region was identified $121 \mathrm{bp}$ upstream from a Shine-Dalgarno region.

In addition to these probable -10 and ShineDalgarno regions, we identified highly homologous sequences of ATGATAAAT and TATAAAAAATCA at about 2 and $21 \mathrm{bp}$ downstream of the probable -10 region of the $C$. butyricum, $C$. botulinum type $\mathrm{E}$ and tetanus toxin genes, and the sequence ATATTAAA was found between the probable promoter and ShineDalgarno regions in all of the C. butyricum, type A, type $\mathrm{C}$, type $\mathrm{E}$ and tetanus toxin genes. We propose that these homologous sequences may play a role in regulation of expression of the toxin genes.

It has been reported that tetanus toxin and the different types of botulinum toxins possess several consensus amino acid sequences of between 7 and 20 residues in length (Tsuzuki et al., 1988). In addition to these sequences, we found three other highly homologous regions in botulinum type $\mathrm{A}, \mathrm{C}, \mathrm{E}$, butyricum and tetanus toxins (Fig. 4). The nucleotide sequences corresponding to these three homologous regions were also quite similar to each other (Fig. 3). One region (amino acid residues 65 to 85 in butyricum toxin) was hydrophilic, and another, the region 201-223, was hydrophobic. This hydrophobic region might conceivably play a role in spanning the mammalian cell membrane, as suggested for tetanus toxin by Eisel $e t$ al. (1986).

Detection of toxin gene in toxigenic and nontoxigenic strains of C. butyricum

DNA samples $(2,20$, and $40 \mu \mathrm{g})$ from one toxigenic and nine nontoxigenic strains of $C$. butyricum were spotted on a nylon membrane, and the membrane was hybridized with the $1 \mathrm{kbp}$ EcoRI probe from the C. butyricum toxin 


\begin{tabular}{|c|c|c|c|c|c|c|c|c|}
\hline Type A & AAACCCACTT & ACAAAATTAT & TTCACACAAT & TTATTCAACT & CATCTTCCAA & CAATCTTSTS & AACATCAATA & \\
\hline Type C & AGAAATTATA & AACTTATTTA & AAAGAATTAA & TTCTACACAA & ATACCAGAAC & AATTAATATA & TACACTTTCC & \\
\hline Tetanus & ACAAACCATC & CTAAAACTCT & TTAACAGAAT & TAAAAACAAT & CTAGCACCTC & AACCCTTATT & ACATAAGATA & \\
\hline & & & & & & & & 630 \\
\hline Butyricum & CCATATTTAC & CAAATCATAA & TACTCCACAT & COTCACTTCA & TTATTAATGA & TCCATCACCA & GTTCCAATTC & \\
\hline Type E & 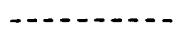 & $-G------$ & $\cdots+\cdots$ & $A A-C-A--C$ & $A-\cdots G C-\cdots$ & $-\cdots-\cdots$ & $---C A C---A$ & \\
\hline Type A & GTAAGGCGAA & TACCATTTTG & CGCTCGAACT & ACAATACATA & CACAATTAAA & ACTTATTCAT & ACTAATTCTA & \\
\hline Type C & ACACATATAC & CCTTTCCTCG & CAATAACAAT & ACTCCAATTA & ATACTTTTCA & TTTTCATCTA & CATTTTAACA & \\
\hline Tetanus & ATAAATCCCA & TACCTTACCT & TCGAAATTCA & TATTCCTTAC & TACACAACTT & TCATACAAAC & TCTAATTCAC & \\
\hline & & & & & & & & 700 \\
\hline Butyricum & AATTCTCAAA & TGCTACCCAA & ACCATACTAT & TACCTAATCT & TATTATAATC & CCACCACACC & CTCATTTATT & \\
\hline Type E & $\cdots$ & $\cdots$ & CA $\cdots \cdots$ & 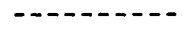 & $\cdots$ & $\cdots$ & $\cdots-\cdots$ & \\
\hline Type A & TTAATGTCAT & ACAACCAGAT & CGTAGTTATA & CATCAGAACA & ACTTAATCTA & CTAATAATAC & GACCCTCACC & \\
\hline Type C & CTCTTCATCT & TAAAACTACA & CAACCTAACA & ACTCGCTTAA & AACTCCTACC & ATAAATCCTA & CTCTTATAAT & \\
\hline Tetanus & TATCTTTTAA & TTTATTAGAA & CAACACCCCA & CTCCACCAAC & TACAAAATCA & CCAATCCTCA & CAAATTTAAT & \\
\hline & & & & & & & & 770 \\
\hline Butyricum & TCAAACTAAC & ACTTCCAATA & TTICTCTAAC & AAATAATTAT & $\triangle$ ICCCAACCA & ATCACCCTTT & TCCATCAATA & \\
\hline Type E & 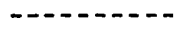 & $\cdots$ & 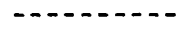 & 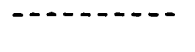 & -------- & $--1+-1$ & $\cdots$ & \\
\hline Type A & TGATATTATA & CACTTTCAAT & CTAAAACCTT & IGCACATCAA & CTTTTCAATC & ITACCCCAAA & TCCTTATCCC & \\
\hline Type C & AACTCCACCT & $A C A G A A A A C A$ & TTATACATCC & ACAAACTTCT & АCCTTTAAAT & TAACTAACAA & TACTTTTCCC & \\
\hline Tetanus & AATATTTCCA & CCTCCCCCTC & TTTTAAATAA & AAATCACCTT & ACACCTATTC & TATTCACCCT & ACATAATAAA & \\
\hline & & & & & & & & 840 \\
\hline Butyricum & CCTATACTAA & CAITCTCACC & TCAATATTCT & ITIACATTTA & AACATAATAC & TATCAATCAA & TTTATTCAAG & \\
\hline Type E & - & 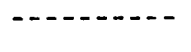 & 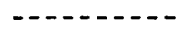 & $\cdots$ & $-\mathrm{T}-\ldots-\cdots$ & $\cdots$ & $\cdots$ & \\
\hline Type A & TCTACTCAAT & ACATTACATT & TACCCCACAT & TITACATTTC & CTTTTCACCA & CTCACTTCAA & CTTCATACAA & \\
\hline Type C & CCACAACAAC & CATTICCTCC & TTTATCAATA & ATTTCAATAT & CACCTACATT & TATCCTAACA & TATACTAATC & \\
\hline Tetanus & AATTACTTCC & CATCTACACA & TCCTTTICCC & TCAATAATCC & AAATCCCATT & TTCCCCACAA & TATCTACCTA & \\
\hline & & $\Gamma \wedge$ & & & & & & 910 \\
\hline Butyricum & ATCCTCCTCT & TACATTAATC & CATCAATTAA & TACATTCATT & ACATCCACTA & TATCCCCCTA & AAGCCATTAC & \\
\hline Type E & 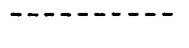 & 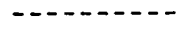 & 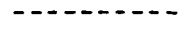 & ------- & 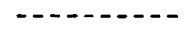 & 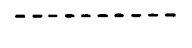 & $\ldots \ldots-\ldots$ & \\
\hline Type A & ATCCTCTTTT & ACGTCCACGC & AAATTTCCTA & CACATCCACC & ACTAACATTA & CCACATCAAC & TTATACATCC & \\
\hline Type C & CAACTAATGA & TCTACCACAC & CCTACATTTT & CTAACTCTCA & ATTTTCCATC & CATCCAATAC & TAATTTTAAT & \\
\hline Tetanus & CCTTTGATAA & TCTAATAGAA & AATATTACGT & CACTCACTAT & TCCCAAAACC & AAATATTTTC & AACATCCACC & \\
\hline Butyricum & TACAATCTAT & ACTATAACAC & AAAAACAAAA & TCCCCTAATA & ACAAATATAA & CACGTACAAA & TATTCAACAA & ITC \\
\hline Type E & $\cdots+-\cdots+\cdots$ & $-\cdots---$ & 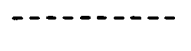 & - - - & 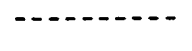 & $\cdots+-\cdots$ & 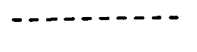 & $-\cdot$ \\
\hline Type A & TCCACATACA & TTATATCCAA & TACCAATTAA & TCCAAATACC & GITTTIAAAC & TAAATACTAA & TCCCTATTAT & CAA \\
\hline Type C & GCATCAACTT & AATCATCCAA & TCCATAATTT & ATATCCAATA & CCTATACCAA & ATCATCAAAC & AATTTCATCT & CTA \\
\hline Tetanus & ATTACTATTA & ATCCACGAAC & TTATACATCT & ACTACATCCT & TTATACCCAA & TCCACCTATC & AACCCATCAA & ATT \\
\hline
\end{tabular}

Fig. 3. Nucleotide sequences of the 5'-translated and 5'-untranslated regions of butyricum, botulinum and tetanus toxin genes. The DNA sequence of the butyricum toxin gene EcoRI fragment is compared to the sequence of botulinum type A (Binz et al., 1990), type C (Kimura et al., 1990), type E (Fujii et al., 1990) and tetanus (Eisel et al., 1986) toxin genes. The putative Shine-Dalgarno sequence is indicated by SD. The sequences homologous to the -10 region of the $E$. coli promoter (TATAAT) are marked with double underlines. A sequence with similar dyad symmetry is underlined by divergent arrows. Numbers represent free energy values in kcal mol-1 $(1 \mathrm{kcal}=4.2 \mathrm{~kJ})$. Dashes in the botulinum type $\mathrm{E}$ sequence represent bases identical to the $C$. butyricum sequence. The highly homologous sequences are underlined, and the start site of homology is indicated on the butyricum sequence by A (type A), C (type C) and $T$ (tetanus). ORF is the translation initiation site of ATG. 


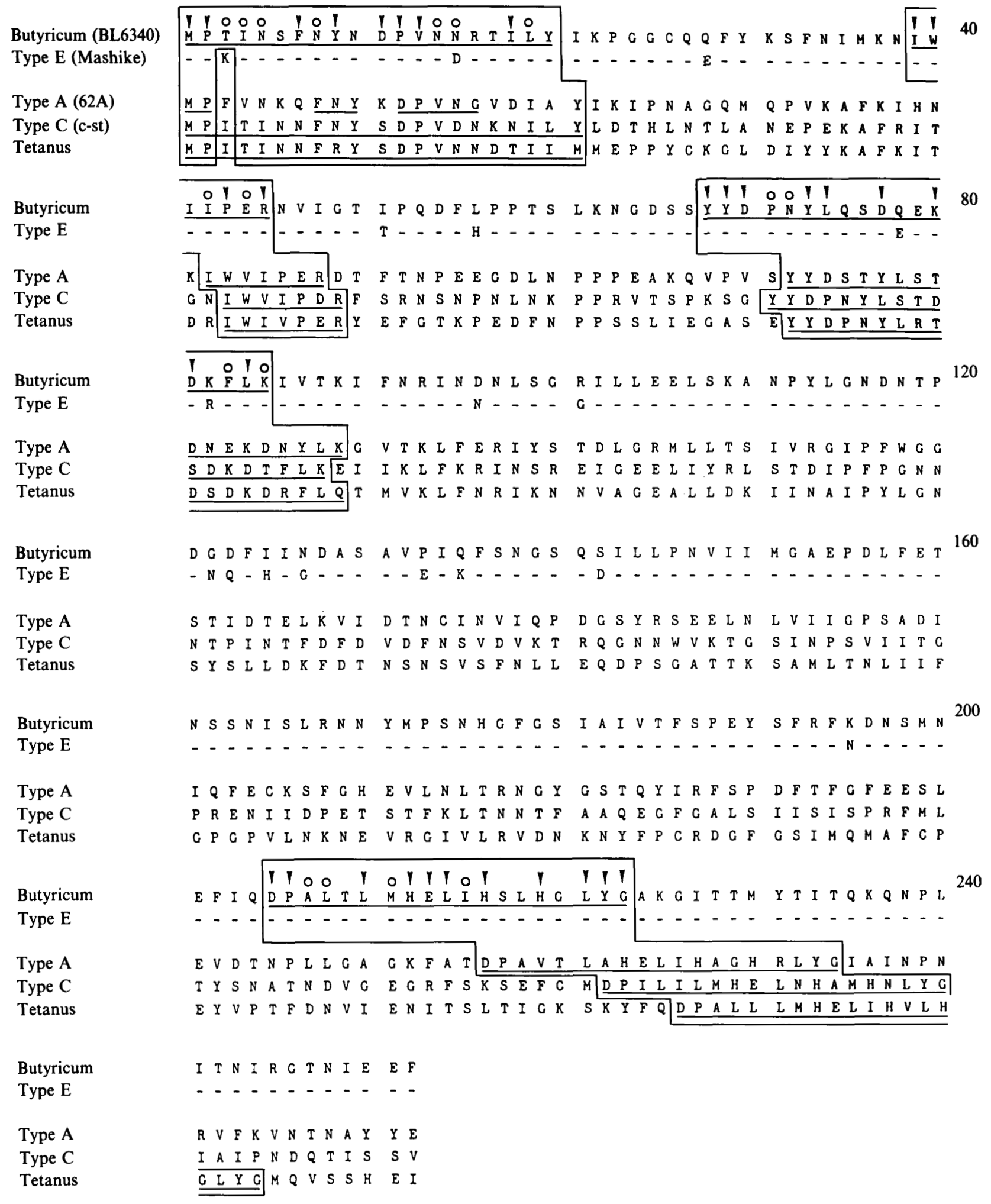

Fig. 4. Amino acid sequences derived from the base sequence of butyricum toxin gene EcoRI fragment. Dashes in the botulinum type $\mathrm{E}$ sequence represent amino acid residues identical to the $C$. butyricum sequence. The highly conserved homologous sequences are underlined. Arrows indicate highly conserved amino acid residues among the five toxins, and open circles indicate common amino acid residues in four out of five toxins. 
gene. A positive reaction was observed only with DNA from the toxigenic strain, BL6340 (data not shown). The nucleotide sequences of the toxin genes from $C$. butyricum and $C$. botulinum strains were quite similar in both coding and noncoding regions. Therefore, it is quite possible that a nontoxigenic $C$. butyricum strain has become toxigenic by acquiring the toxin gene from a $C$. botulinum type $\mathrm{E}$ strain; the toxin gene could perhaps have been transmitted from $C$. botulinum type $\mathrm{E}$ to $C$. butyricum cells through some transmissible vector, e.g. a bacteriophage or a plasmid.

This study was supported by grants from the Ohyama Health Foundation and the Mishima Memorial Foundation.

\section{References}

Betlay, M. J., Somers, E. \& DasGupta, B. R. (1989). Characterization of botulinum type A neurotoxin gene: Delineation of the Nterminal encoding region. Biochemical and Biophysical Research Communications 162, 1388-1395.

Binz, T., Kurazono, H., Wille, M., Frevert, J., Wernars, K. \& NiEMANN, H. (1990). The complete sequence of botulinum neurotoxin type $A$ and comparison with other clostridial neurotoxins. Journal of Biological Chemistry 256, 9153-9158.

Eisel, U., Jarausch, W., Goretzki, K., Henschen, A., Engels, J., Weller, U., Hudel, M., Hakermann, E. \& Niemann, H. (1986). Tetanus toxin: primary structure, expression in $E$. coli, and homology with botulinum toxins. EMBO Journal 5, 2495-2502.

FuiII, N., Oguma, K., Yokosawa, N., Kimura, K. \& Tsuzuki, K. (1988). Characterization of bacteriophage nucleic acids obtained from Clostridium botulinum types C and D. Applied and Environmental Microbiology 54, 69-73.

FujI, N., Kimura, K., Indoh, T., Murakami, T., Yashiki, T., Tsuzuki, K., Yokosawa, N. \& Oguma, K. (1990). The nucleotide and deduced amino acid sequences of EcoRI fragment containing the 5'-terminal region of Clostridium botulinum type E toxin gene cloned from Mashike, Iwanai and Otaru strains. Microbiology and Immunology 34, 1041-1047.

Gimenez, J., Foley, J. \& DasGupta, B. R. (1988). Neurotoxin type E from Clostridium botulinum and C. butyricum; Partial sequence and comparison. FASEB Journal 2, A1750(8447).

Hall, J. D., McCroskey, L., Pincomb, B. J. \& Hatheway, C. L. (1985). Isolation of an organism resembling Clostridium barati which produces type $\mathrm{F}$ botulinal toxin from an infant with botulism. Journal of Clinical Microbiology 21, 654-655.

Kimura, K., Funil, N., Tsuzuki, K., Murakami, T., Indoh, T., YokosawA, N. \& OGUMA, K. (1990). The complete nucleotide sequence of the gene coding for botulinum type $\mathrm{C}_{1}$ toxin in the c-st phage genome. Biochemical and Biophysical Research Communications 171, 1304-1311.

McCroskey, L. M., hatheway, C. L., Fenicia, L., Pasolini, B. \& AURELI, P. (1986). Characterization of an organism that produces type E botulinal toxin but which resembles Clostridium butyricum from the feces of an infant with type E botulism. Journal of Clinical Microbiology 23, 201-202.

MARMUR, J. (1961). A procedure for the isolation of deoxyribonucleic acid from micro-organisms. Journal of Molecular Biology 3, 208-218.

Morishi, K., SyUto, B., Kubo, S. \& OGUMA, K. (1989). Molecular diversity of neurotoxins from Clostridium botulinum type D strains. Infection and Immunity 57, 2886-2891.

SathYAMOORTHY, V. \& DASGUPTA, B. R. (1985). Separation, purification, partial characterization and comparison of heavy and light chains of botulinum neurotoxin types A, B, and E. Journal of Biological Chemistry 260, 10461-10466.

Suen, J. C., Hatheway, C. L., Steigerwalt, A. G. \& Brenner, D. J. (1988). Genetic confirmation of identities of neurotoxigenic Clostridium barati and Clostridium butyricum implicated as agents of infant botulism. Journal of Clinical Microbiology 26, 2191-2192.

Thompson, E. D., Brehm, K. J., Oultram, D. J., Swinfield, T. J., Shone, C. C., Atkinson, T., Melling, J. \& Minton, P. N. (1990). The complete amino acid sequence of the Clostridium botulinum type A neurotoxin, deduced by nucleotide sequence analysis of the encoding gene. European Journal of Biochemistry 189, 73-81.

Tsuzuki, K., Yokosawa, N., SYuto, B., OHISHI, I., FuiI, N., Kimura, K. \& OGUmA, K. (1988). Establishment of a monoclonal antibody recognizing an antigenic site common to Clostridium botulinum type $\mathrm{B}, \mathrm{C}_{1}, \mathrm{D}$ and $\mathrm{E}$ toxins and tetanus toxin. Infection and Immunity 56, 898-902. 